cause, and mere necessary machinery, that so much of the barren disputes of biology are due," wrote Huxley. It would seem that, with such a sloppy and essentially misguided interpretation, these disputes would not be solved in a hurry. Unfortunately, even Tinbergen's careful analysis of cause and function could not prevent a confusion of concepts that continues to this day.

Some will say that ethology is no longer a scientific discipline in its own right, but that depends on who you ask: a behavioural ecologist and a cognitive ethologist might give you different answers. Nevertheless, Burkhardt notes that the core ideas of classical ethology dissipated astonishingly rapidly; few contemporary ethologists would use such concepts as 'action-specific energy', for example. This discarding of outmoded ideas would seem natural for any vibrant scientific discipline.

Burkhardt rightly maintains that it was the empirical and theoretical approach introduced by Lorenz, Tinbergen and their colleagues that made the study of animal behaviour what it is today. In order to study the genomic or neural mechanisms of behaviour, we need to know how behaviour works, and for that an ethological analysis is crucial. This wonderful book shows very clearly how early ethologists made such analysis possible.

Johan J. Bolhuis is in the Department of Biology,

Utrecht University, Padualaan 14,

3584 CH Utrecht, The Netherlands.

\title{
DANCE
}

\section{Einstein in motion}

\section{Constant Speed \\ Rambert Dance Company \\ At Sadler's Wells, London, 24-28 May \\ 2005. UK tour begins September 2005. \\ www.rambert.org.uk}

\section{Alison Wright}

I admit, I cringed when I heard that the UK Institute of Physics (IOP) had commissioned a ballet to celebrate the World Year of Physics 2005, or 'Einstein Year'. Truly enlightening meetings of art and science are rare indeed. Einstein: The Ballet? Please, no.

But the IOP had wisely put themselves in the hands of the Rambert Dance Company, and the results, revealed at a Sadler's Wells première on 24 May, are stunning.

Constant Speed is inspired by Einstein's 1905 publications; the World Year of Physics celebrates their centenary. From the patent office in Bern, Switzerland, Einstein dashed off five papers, all of them seminal work, on three themes: brownian motion, the photoelectric effect and the special theory of relativity.

Relativity, and $E=m c^{2}$, will be forever associated with Einstein. But it is his work on the photoelectric effect, which established the notion of the 'quantum', that Einstein himself regarded as his most revolutionary. The effect describes the release of electrons from a metal when light is shone on its surface. To explain the relation between the energy of the electrons released and the frequency of the incident light, Einstein proposed that light energy is transferred to the electrons in distinct chunks, or quanta. No less significant was his study of fluctuation phenomena within the framework of kinetic-molecular theory - work that recalled the brownian motion seen in the dance of pollen grains in water decades earlier.

Choreographer Mark Baldwin, the artistic director of the Rambert, developed Constant Speed through conversations with Ray Rivers, professor of theoretical physics at Imperial College London. Although he claims to be ignorant of physics, Baldwin was struck by a similarity of language - space, time, energy between physics and dance. Quite rightly, I

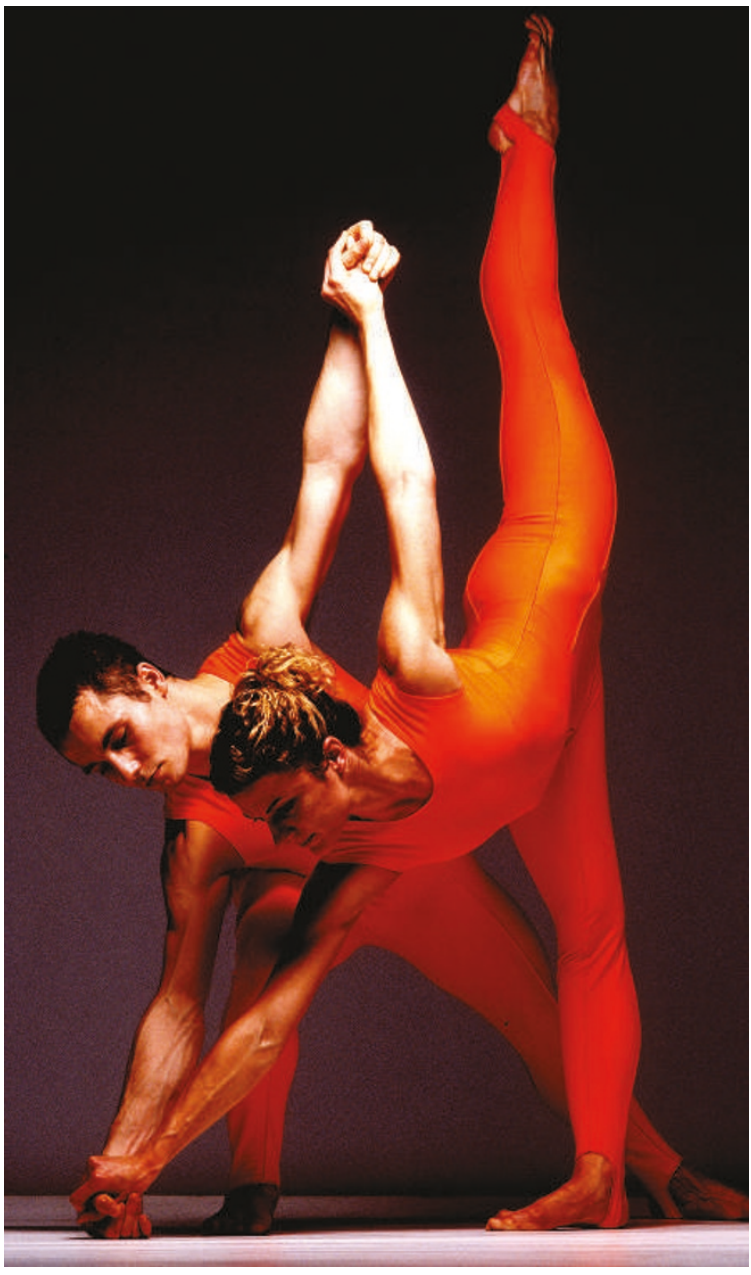

Getting physical: dancers of the Rambert Dance Company.

metallic stage is bathed in light. Or they could be photons, tight packets of energy that also have concerted, wave-like motions. Then the whiteness is subsumed by red, with red lighting and red costumes as more dancers join the fray, evolving eventually through the full spectrum of colour. The colour-coded dancers are compartmentalized - quantized, if you like - each pair exploring its own motion at its own frequency until the rainbow finale. The music by Franz Lehár, composed like Einstein's physics in 1905, adds another dimension: you can almost imagine Einstein day-dreaming, seeing the multicoloured quanta of his theory before him as he hummed along to the pop music of his day.

At just 27 minutes long, the swirling colour of Constant Speed seemed to be over all too quickly. But the work was superbly complemented by the preceding programme, especially Momenta, created by Rambert dancer Mikaela Polley. To music by Patrick Nunn, the dance builds steadily in energy, speed and coherence (more physics!). Different moods were created by the tawdry humour of the opening piece, Judgement of Paris, and the heart-rending emotion of Dark

think, he shied away from specifically representing relativity in the piece, alluding only in the title to that theory's central tenet of a constant speed of light. Brownian motion, on the other hand, is a concept easily reflected in the movements and configuration of the dancers. But it is the ideas surrounding the photoelectric effect that dominate Constant Speed.

Running on to the stage, fists clenched and elbows pumping, the white-clad dancers could be photoelectrons, released as the seemingly
Elegies. However, the maturity of these pieces, choreographed by Anthony Tudor in the 1930s, perhaps highlighted a few rough edges in Constant Speed.

The physics is there if you look - but don't look too hard. As Baldwin said, this is not a physics lesson. Rather, Constant Speed is about inspiration: inspiring physics and inspired dance.

Alison Wright is the editor of Nature Physics.

www.nature.com/naturephysics 